\title{
High-resolution numerical approximation of traffic flow problems with variable lanes and free-flow velocities
}

\author{
Peng Zhang, ${ }^{1, *}$ Ru-Xun Liu, ${ }^{1}$ and S. C. Wong ${ }^{2}$ \\ ${ }^{1}$ Department of Mathematics, University of Science and Technology of China, Hefei, Anhui, People's Republic of China \\ ${ }^{2}$ Department of Civil Engineering, The University of Hong Kong, Hong Kong SAR, People's Republic of China
}

(Received 3 November 2003; revised manuscript received 6 July 2004; published 20 May 2005)

\begin{abstract}
This paper develops macroscopic traffic flow models for a highway section with variable lanes and free-flow velocities, that involve spatially varying flux functions. To address this complex physical property, we develop a Riemann solver that derives the exact flux values at the interface of the Riemann problem. Based on this solver, we formulate Godunov-type numerical schemes to solve the traffic flow models. Numerical examples that simulate the traffic flow around a bottleneck that arises from a drop in traffic capacity on the highway section are given to illustrate the efficiency of these schemes.
\end{abstract}

DOI: 10.1103/PhysRevE.71.056704

PACS number(s): 02.70.-c, 45.70.Vn, 83.85.Pt

\section{INTRODUCTION}

Since the development by Lighthill and Whitham [1] and independently by Richards [2], of the traffic flow model, which is now known as the LWR model, continuum traffic flow models have been intensively studied (see [3-23]). All of these models incorporate the "standard" conservative laws of flux in the form of $f(u(x, t))$, for which it is not difficult to develop numerical schemes. However, the number of lanes on a highway frequently changes, which generally results in spatially varying flux, but the traffic flow literature contains few works on this area of research. Helbing [24-26] derived a macroscopic model of mixed multilane freeway traffic using a gas-kinetic approach. Newell [27] developed a model with continuously varying spatial parameters, in which the capacity is a quadratic function of the location variable.

However, for a discontinuous variation in the number of lanes (and in the free-flow velocity for the more general case in this paper), the problem is very complicated, and is usually nonstrictly hyperbolic. In general, the problem of variable lanes (and free-flow velocities) can be characterized by flux of the form $f(u(x, t), a(x))$. In such cases, the schemes that are designed for the approximation of flux $f(u(x, t))$ are not efficient because they cannot handle the discontinuity that is caused by the change in $a(x)$. Experience has found that numerical instability may occur, especially when $a(x)$ has a sharp gradient $[23,28-32]$. Although the instability may be avoided by using sufficiently refined mesh and higherorder schemes, such as the Runge-Kutta discontinuous Galerkin (RKDG) scheme [33], the solution may sometimes not converge to one that is physically relevant. In fact, the theories of weak solutions and the corresponding numerical methods for this kind of problem are still under investigation (see Refs. [30-32,34-37] for useful discussions in general and on other applications).

This paper has two main objectives. The first is to extend the LWR model $[1,2]$ and the higher-order model to cases of

\footnotetext{
*Corresponding author. E-mail addresses: Pengzhang@ustc.edu, Pzhang@mail.shu.edu.cn
}

variable lanes and free-flow velocities. The extension of the former is straightforward, whereas the relaxation equation of the latter is derived through comparing its acceleration with that of the extended LWR model. The second objective is to develop an exact Riemann solver that encapsulates the spatially varying flux functions, based on which the Godunovtype numerical schemes will be formulated to solve the traffic flow models.

The reminder of the paper is organized as follows. Section II presents the formulation of the traffic flow models. Section III derives the Riemann solver for the problem. Section IV formulates the Godunov-type numerical schemes. Section V gives numerical examples for the models.

\section{FORMULATION OF THE MODELS}

As in the LWR theory, the traffic flow is considered as a compressible fluid. Let $a(x)$ be a variable that represents the number of lanes on a highway. The vehicle conservation equation can be written as

$$
(a \rho)_{t}+(a \rho u)_{x}=0, \quad x \in R \equiv(-\infty,+\infty), \quad t \in R_{t}^{+} \equiv[0, \infty),
$$

where $\rho(x, t)$ is the density in a traffic lane, which is expressed in number of vehicles per unit length of the highway per lane, and $u(x, t)$ is the average speed, which is identical across adjacent lanes, at $(x, t)$.

\section{A. Extended LWR model}

At location $x$, the total density (number of vehicles per unit length) is $a \rho$ with a maximum of $a \rho_{m}$, where $\rho_{m}$ is the jam density of $\rho$ on a lane. The LWR model assumes a strictly decreasing function of $u$ in $a \rho$, which is known as the speed-density relationship. More precisely, it is a monotone curve from $(0, b)$ to $\left(a \rho_{m}, 0\right)$ in the $(a \rho)$ - $u$ coordinate plane, where $b$ is the free-flow speed (i.e., the maximum of $u$ ) locally at $x[b=b(x)]$. By scaling, the speed $u$ is a strictly decreasing function of $\rho$, i.e., 


$$
u=u_{e}(\rho, b),
$$

that satisfies $u_{e}(0, b)=b$ and $u_{e}\left(\rho_{m}, b\right)=0$. Combining Eqs. (1) and (2), we have a closed equation, which is called the extended LWR (ELWR) model with variable lanes, and can be written as

$$
(a \rho)_{t}+\left(a \rho u_{e}(\rho, b)\right)_{x}=0 .
$$

\section{B. Extended higher-order model}

In this section, we develop an extended higher-order (EHO) model. The formulation is based on a mechanism that is similar to the so called "look-forward" mechanism of a driver (see Payne and Whitham [3,4] and Zhang [17]); the comparison of the acceleration with that of the ELWR model; and the introduction of a fluctuation term that simulates the stop-and-go waves.

We first derive the traffic acceleration of the ELWR model in a way that is similar to that of Pipes [38]. By the continuity equation (1), we have

$$
\rho_{t}+u \rho_{x}=-\rho u_{x}-\frac{\rho u}{a} a^{\prime}, \text { or } \rho_{t}+u \rho_{x}+\frac{\rho u}{a} a^{\prime}=-\rho u_{x} .
$$

We replace $u$ in Eq. (4) with $u_{e}(\rho, b)$, and thus

$$
\rho_{t}+u_{e} \rho_{x}=-\rho \frac{\partial u_{e}}{\partial \rho} \rho_{x}-\rho \frac{\partial u_{e}}{\partial b} b^{\prime}-\frac{\rho u_{e}(\rho) a^{\prime}}{a} .
$$

We take the total derivative of Eq. (2) with respect to $t$, which yields

$$
\frac{d u}{d t}=\frac{\partial u_{e}}{\partial \rho}\left(\rho_{t}+\frac{d x}{d t} \rho_{x}\right)+\frac{\partial u_{e}}{\partial b} \frac{d x}{d t} b^{\prime}=\frac{\partial u_{e}}{\partial \rho}\left(\rho_{t}+u_{e} \rho_{x}\right)+\frac{\partial u_{e}}{\partial b} u b^{\prime},
$$

where $d x / d t=u=u_{e}(\rho)$. Comparing these two expressions, we obtain

$$
\frac{d u}{d t}=-\rho\left(\frac{\partial u_{e}}{\partial \rho}\right)^{2} \rho_{x}+\left(u-\rho \frac{\partial u_{e}}{\partial \rho}\right) \frac{\partial u_{e}}{\partial b} b^{\prime}-\frac{\rho u}{a} \frac{\partial u_{e}}{\partial \rho} a^{\prime} .
$$

We then consider the "look-forward" effect. In Refs. $[3,4,17], a(x)$ and $b(x)$ are constant, and this effect is considered according to the relation $u(x(t+\tau), t+\tau))=u_{e}(\rho(x$ $+\Delta x, t)$ ), in which the driver makes a decision after a time lag $\tau$ (or relaxation time) in response to the traffic condition downstream at location $x+\Delta x$. However, this dependency is not suitable for our case, because the velocity may vary greatly with locations due to a sharp change in the traffic capacity that is described by the functions $a(x)$ and $b(x)$. In addition, it is well known that the velocity and density are usually discontinuous because of wave breaking, whereas the flux is always continuous (or varies less sharply) in a hyperbolic system. Therefore, to consider the "look-forward" effect, we replace the velocity in the above with the flux according to the relation

$$
\begin{aligned}
a(x) u(x, t+\tau) \rho(x, t+\tau)= & a(x+\Delta x) u_{e}(\rho(x+\Delta x, t), b(x+\Delta x)) \\
& \times \rho(x+\Delta x, t),
\end{aligned}
$$

where on the left-hand side $x=x(t+\tau)$. We take the Taylor series expansions in $\tau$ on the left-hand side (LHS), and in $\Delta x$ on the right-hand side (RHS), respectively, as

$$
\text { LHS }=a u \rho+\tau\left[a \rho \frac{d u}{d t}+a u\left(\rho_{t}+u \rho_{x}+\frac{\rho u}{a} a^{\prime}\right)\right],
$$

and

$$
\mathrm{RHS}=a u_{e} \rho+\Delta x\left[a \rho\left(\frac{\partial u_{e}}{\partial \rho} \rho_{x}+\frac{\partial u_{e}}{\partial b} b^{\prime}\right)+a u_{e} \rho_{x}+\rho u_{e} a^{\prime}\right] .
$$

By the comparison of Eq. (4) and LHS=RHS, it is easy to obtain

$$
\frac{d u}{d t}=\frac{u_{e}-u}{\tau}+\frac{\Delta x}{\tau}\left(\frac{\partial u_{e}}{\partial \rho} \rho_{x}+\frac{\partial u_{e}}{\partial b} b^{\prime}+\frac{u_{e}}{a} a^{\prime}+\frac{u_{e}}{\rho} \rho_{x}\right)+u u_{x} .
$$

Zhang [17] chose the quantity $\Delta x$ that is consistent with the acceleration expression that is derived from the LWR model. Similarly, we choose $\Delta x$ in such a way that the acceleration expressions $d u / d t$ in Eqs. (5) and (6) are identical when setting $u=u_{e}(\rho, b)$. This can be realized by choosing

$$
\Delta x=-\tau \rho \frac{\partial u_{e}}{\partial \rho}
$$

which is exactly the same as in Zhang [17]. We must handle the last term of Eq. (6) appropriately, for which $u$ is approximated by $u_{e}$ under the same consideration. This gives the following description of the acceleration:

$$
\begin{aligned}
u_{t}+u u_{x}= & \frac{u_{e}-u}{\tau}-\rho\left(\frac{\partial u_{e}}{\partial \rho}\right)^{2} \rho_{x}+\left(u_{e}-\rho \frac{\partial u_{e}}{\partial \rho}\right) \frac{\partial u_{e}}{\partial b} b^{\prime} \\
& -\frac{\rho u_{e}}{a} \frac{\partial u_{e}}{\partial \rho} a^{\prime} .
\end{aligned}
$$

Note that $d u / d t$ is identical to $u_{t}+u u_{x}$.

Due to its relation (consistency) with the ELWR model, which is well-posed so that the Riemann solution is existent and unique (see Sec. III), this acceleration describes a traffic state that is very close to equilibrium flow. Actually, its smooth solution converges to that of the ELWR model, namely, $u \rightarrow u_{e}(\rho, b)$ (Sec. IV C). This convergence also applies to the case of weak solutions if the conservation form of Eq. (8) is consistent with the continuity equation (1) (Sec. IV B). These arguments are also confirmed numerically (Sec. $\mathrm{V})$. For the special case in which $a(x)$ and $b(x)$ are constant throughout the highway section, Eq. (8) reduces to that of Zhang [17], in which case the same relation with the LWR model is indicated numerically [18]. It can be understood that the description of Eq. (8) is a dynamic version of the ELWR model, and that it possesses favorable stable and convergent properties.

However, some important traffic flow features are missing, such as phase transitions and stop-and-go waves (see Refs. [13,14,39] for details). As an attempt to simulate a traffic flow that is in a so called metastable state, we introduce a fluctuation term, which is added to Eq. (8) to give the following EHO model: 


$$
\begin{aligned}
u_{t}+u u_{x}= & \frac{u_{e}-u}{\tau}+\frac{\tilde{u}_{e}-u_{e}}{\tilde{\tau}}-\rho\left(\frac{\partial u_{e}}{\partial \rho}\right)^{2} \rho_{x}+\left(u_{e}-\rho \frac{\partial u_{e}}{\partial \rho}\right) \frac{\partial u_{e}}{\partial b} b^{\prime} \\
& -\frac{\rho u_{e}}{a} \frac{\partial u_{e}}{\partial \rho} a^{\prime} .
\end{aligned}
$$

$\widetilde{u}_{e}=\widetilde{u}_{e}(\rho, b)$ is another velocity-density relation that is supposed to share the same free-flow velocity as $u_{e}(\rho, b)$. The first two terms on the right-hand side can be merged,

$$
\frac{u_{e}-u}{\tau}+\frac{\tilde{u}_{e}-u_{e}}{\tilde{\tau}}=\frac{U_{e}-u}{\tau}, \quad U_{e}=\frac{\tau}{\tilde{\tau}} \widetilde{u}_{e}+\left(1-\frac{\tau}{\tilde{\tau}}\right) u_{e}, \quad \tau \leqslant \widetilde{\tau} .
$$

$U_{e}$ is supposed to be a convex combination of $u_{e}$ and $\tilde{u}_{e}$, and is also a velocity-density relation. Thus, the new merged term can be viewed as a relaxation of $u$ to $U_{e}$.

In Eq. (9), the factors for stability and fluctuation are combined so that the traffic state in between will be described, which is known as metastability. It is obvious that the stability decreases if the weight $\tau / \widetilde{\tau}$ increases. In the sections that follow, the fundamental properties of the ELWR and EHO models will be further revealed. Our main objective is to develop a high-resolution scheme for the approximation of such complicated systems, so that the relevant propagation information may be correctly captured.

In the forthcoming section, the Riemann problem is solved to obtain the exact numerical flux at the interface. The discussion is limited to scalar equation (3), or the ELWR model, because the defined conservation form of the EHO model takes similar convective parts (Sec. IV) that can be approximated in the same way.

\section{RIEMANN PROBLEM AND NUMERICAL FLUX}

For simplicity, we assume $b(x)$ to be constant. For the general case in which $b^{\prime}(x) \neq 0$, the wave breaking of the Riemann problem is obviously the same pattern, and the conclusion is easily extended at the end of this section. Under this assumption, we use the denotation $u_{e}(\rho, b)=u_{e}(\rho)$ and rewrite Eq. (3) as

$$
(a \rho)_{t}+(a f(\rho))_{x}=0, \quad f(\rho)=\rho u_{e}(\rho) .
$$

Note that $f(\rho)$ can be any strictly concave function $\left[f^{\prime \prime}(\rho)\right.$ $<0]$. We denote $\rho^{*}$ as the critical density, so that $f\left(\rho^{*}\right)$ is the maximum of $f(\rho)$, i.e., $f^{\prime}\left(\rho^{*}\right)=0$. Thus, it is obvious that $f(\rho)$ is strictly increasing on $\left[0, \rho^{*}\right]$ and strictly decreasing on $\left[\rho^{*}, \rho_{m}\right]$. With $a(x)$ being discontinuous, the Riemann problem of Eq. (11) can be specified as

$$
\begin{aligned}
& a(x)= \begin{cases}a^{-}, & x \in R^{-} \equiv(-\infty, 0), \\
a^{+}, & x \in R^{+} \equiv(0,+\infty),\end{cases} \\
& \rho_{0}(x) \equiv \rho(x, 0)= \begin{cases}\rho^{-}, & x \in R^{-}, \\
\rho^{+}, & x \in R^{+} .\end{cases}
\end{aligned}
$$

For each domain of $D^{-} \equiv R^{-} \times R_{t}^{+}$and $D^{+} \equiv R^{+} \times R_{t}^{+}$, where $R_{t}^{+}=(0, \infty)$, the problem of wave propagation is well studied [4,40], because $a(x)$ is constant in either domain and Eq. (11) reduces to

$$
\rho_{t}+f(\rho)_{x}=0, \quad(x, t) \in D^{-}\left(\text {or } D^{+}\right) .
$$

Therefore, the main concern is how the two traffic states in $D^{-}$and $D^{+}$connect at their interface, i.e, $x=0$. We assume that $\rho\left(0^{-}, t\right) \equiv \rho_{0}^{-}$and $\rho\left(0^{+}, t\right) \equiv \rho_{0}^{+}$are the density values on the left- and right-hand sides of the interface. First, this connection can be made by the Rankine-Hugoniot jump condition

$$
[a f(\rho)]=[a \rho] \frac{d x}{d t},
$$

which is applicable to any curve in the $x$ - $t$ plane. For the interface (a straight line) $x=0$, in which clearly $d x / d t=0$, we have $[a f(\rho)]=a^{+} f\left(\rho_{0}^{+}\right)-a^{-} f\left(\rho_{0}^{-}\right)$and $[a \rho]=a^{+} \rho_{0}^{+}-a^{-} \rho_{0}^{-}$. The application of Eq. (14) results in

$$
a^{-} f\left(\rho_{0}^{-}\right)=a^{+} f\left(\rho_{0}^{+}\right) .
$$

Clearly, Eq. (15) implies that the in-flow is equal to the outflow at the interface, which satisfies the mass conservation principle. See Refs. [4,40] for details.

Secondly, the two states should be connected by the characteristics, according to the theory that has been proposed by the authors [30]. For a physically relevant solution, the sign of characteristic speeds cannot change across the interface, i.e.,

$$
f^{\prime}\left(\rho_{0}^{-}\right) f^{\prime}\left(\rho_{0}^{+}\right) \geqslant 0 .
$$

Finally, the Lax entropy conditions $[4,40]$ are applied separately in each of the domains $D^{-}$and $D^{+}$, where the "standard" conservation form (13) is applicable. That is, the shock condition in $D^{-}$is given by $f^{\prime}\left(\rho^{-}\right)>f^{\prime}\left(\rho_{0}^{-}\right)$, or $\rho^{-}$ $<\rho_{0}^{-}$, and the shock speed

$$
\frac{d x}{d t} \leqslant 0
$$

Similarly, a shock wave that arises from $f^{\prime}\left(\rho_{0}^{+}\right)>f^{\prime}\left(\rho^{+}\right)$or $\rho_{0}^{+}<\rho^{+}$in $D^{+}$satisfies

$$
\frac{d x}{d t} \geqslant 0
$$

In Eqs. (17) and (18), $d x / d t$ is determined by the jump condition (14).

The constraints (15), (17), and (18) are naturally set, which follow the classical weak solution theory of the equation in the form of Eq. (13). However, with these constraints alone, one can easily construct innumerable solutions for Eq. (11). Therefore, to ensure a unique and physically relevant solution, an extra constraint (16) must be applied. In the following theorem, we will show that the additional constraint (16) is both necessary and sufficient, and that the problem can be solved with $\rho_{0}^{-}$or $\rho_{0}^{+}$[and thus the flux value $a^{-} f\left(\rho_{0}^{-}\right)$or $\left.a^{+} f\left(\rho_{0}^{+}\right)\right]$given at the interface. See Ref. [30] for a more strictly mathematical treatment of the problem.

Theorem. Under conditions (15), (17), and (18), condition (16) is necessary and sufficient for the existence and unique- 


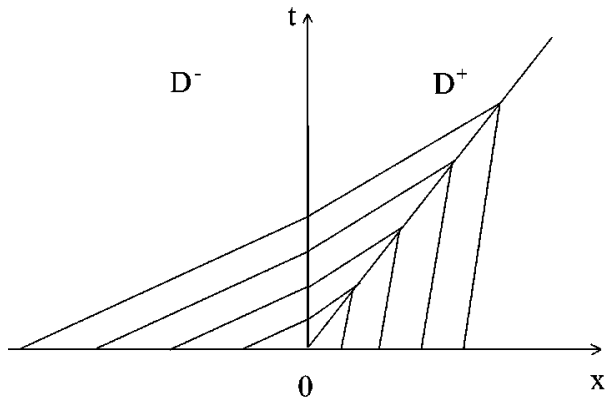

(a)

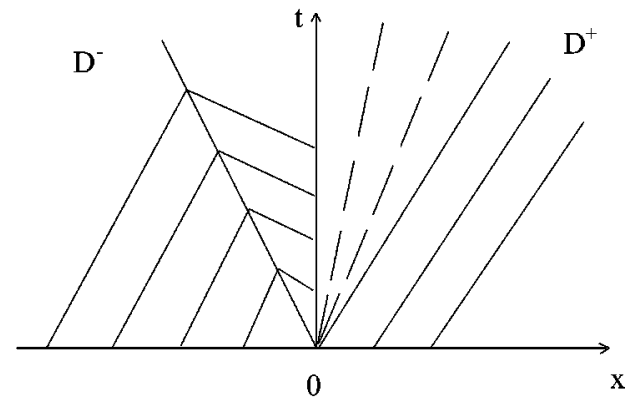

(b)

FIG. 1. Solution structure of the Riemann problem. (a) For $p \in S_{1}$, the propagation of $\rho=\rho^{-}$passes through the interface then changes to $\rho=\rho_{0}^{+}$and forms a shock wave with $\rho=\rho^{+}$in $D^{+}$. (b) For $p \in \bar{S}_{1}$, the propagation of $\rho=\rho^{-}$is obstructed due to the inadequate capacity in $D^{+}$, and shock (bottleneck) and rarefaction waves are formed on the left and right sides of the interface, respectively.

ness of the Riemann solution of Eqs. (11) and (12).

Proof. If condition (16) is removed, then there are multiple solutions that satisfy conditions (15), (17), and (18). Hence, condition (16) is necessary. In the following, we show that Eq. (16) is also sufficient.

Let $P$ be the set of all possible combinations of the parameters of the Riemann problem (12), and $p$ $=\left(a^{-}, \rho^{-}, a^{+}, \rho^{+}\right)$be an element in $P$. The set $P$ can be divided into the following eight subsets:

$$
\begin{aligned}
& S_{1}=\left\{P \mid f^{\prime}\left(\rho^{-}\right)>0, f^{\prime}\left(\rho^{+}\right) \geqslant 0, a^{-} f\left(\rho^{-}\right) \leqslant a^{+} f\left(\rho^{*}\right)\right\}, \\
& \bar{S}_{1}=\left\{P \mid f^{\prime}\left(\rho^{-}\right)>0, f^{\prime}\left(\rho^{+}\right) \geqslant 0, a^{-} f\left(\rho^{-}\right)>a^{+} f\left(\rho^{*}\right)\right\}, \\
& S_{2}=\left\{P \mid f^{\prime}\left(\rho^{-}\right) \leqslant 0, f^{\prime}\left(\rho^{+}\right) \geqslant 0, a^{-} f\left(\rho^{*}\right) \leqslant a^{+} f\left(\rho^{*}\right)\right\}, \\
& \bar{S}_{2}=\left\{P \mid f^{\prime}\left(\rho^{-}\right) \leqslant 0, f^{\prime}\left(\rho^{+}\right) \geqslant 0, a^{-} f\left(\rho^{*}\right)>a^{+} f\left(\rho^{*}\right)\right\}, \\
& S_{3}=\left\{P \mid f^{\prime}\left(\rho^{-}\right) \leqslant 0, f^{\prime}\left(\rho^{+}\right)<0, a^{-} f\left(\rho^{*}\right) \leqslant a^{+} f\left(\rho^{+}\right)\right\}, \\
& \bar{S}_{3}=\left\{P \mid f^{\prime}\left(\rho^{-}\right) \leqslant 0, f^{\prime}\left(\rho^{+}\right)<0, a^{-} f\left(\rho^{*}\right)>a^{+} f\left(\rho^{+}\right)\right\}, \\
& S_{4}=\left\{P \mid f^{\prime}\left(\rho^{-}\right)>0, f^{\prime}\left(\rho^{+}\right)<0, a^{-} f\left(\rho^{-}\right) \leqslant a^{+} f\left(\rho^{+}\right)\right\}, \\
& \bar{S}_{4}=\left\{P \mid f^{\prime}\left(\rho^{-}\right)>0, f^{\prime}\left(\rho^{+}\right)<0, a^{-} f\left(\rho^{-}\right)>a^{+} f\left(\rho^{+}\right)\right\} .
\end{aligned}
$$

Then, we set either $\rho_{0}^{-}$or $\rho_{0}^{+}$at the interface as follows: (a) For $p \in S_{1} \cup S_{4}$ we set $\rho_{0}^{-}=\rho^{-}$, (b) for $p \in \bar{S}_{1} \cup \bar{S}_{2}$, we set $\rho_{0}^{+}$ $=\rho^{*}$, (c) for $p \in S_{3} \cup S_{2}$ we set $\rho_{0}^{-}=\rho^{*}$, (d) for $p \in \bar{S}_{3} \cup \bar{S}_{4}$, we set $\rho_{0}^{+}=\rho^{+}$.

The proofs for cases (d) and (c) are parallel to those of (a) and (b), respectively, and thus we only need to prove cases (a) and (b). In these proofs, we need to verify that (i) $\rho_{0}^{+}$for case (a) $\left[\right.$ or $\rho_{0}^{-}$for case (b)] is uniquely determined by Eqs. (15) and (16), (ii) any shock wave that arises in $D^{-}$or $D^{+}$ satisfies Eqs. (17) or (18), and (iii) Eq. (17) or (18) is violated for any other pair $\left(\rho_{0}^{-}, \rho_{0}^{+}\right)$that satisfies Eqs. (15) and (16), or that this pair produces the same solution, as is shown in the two trivial cases that follow.

For case (a), by Eq. (16) we have $f^{\prime}\left(\rho_{0}^{+}\right) \geqslant 0$, or $\rho_{0}^{+}$ $\in\left[0, \rho^{*}\right]$. To see that $\rho_{0}^{+}$is uniquely determined, we set $q(\rho)=a^{+} f(\rho)-a^{-} f\left(\rho^{-}\right)$, and verify that $q(\rho)$ has just one root. This is obvious, because $q(0)=-a^{-} f\left(\rho^{-}\right) \leqslant 0, q\left(\rho^{*}\right)=a^{+} f\left(\rho^{*}\right)$ $-a^{-} f\left(\rho^{-}\right) \geqslant 0$, and $q^{\prime}(\rho)=a^{+} f^{\prime}(\rho)>0$ for $\rho \in\left(0, \rho^{*}\right)$. Moreover, a shock wave is possible only in $D^{+}$with $\rho_{0}^{+}<\rho^{+}$(the Lax entropy condition), and the shock speed is given by

$$
\frac{d x}{d t}=\frac{f\left(\rho^{+}\right)-f\left(\rho_{0}^{+}\right)}{\rho^{+}-\rho_{0}^{+}} .
$$

If $p \in S_{1}$ [see Fig. 1(a)], then $f^{\prime}\left(\rho^{+}\right) \geqslant 0$, and thus $f\left(\rho_{0}^{+}\right)$ $<f\left(\rho^{+}\right)$. If $p \in S_{4}$, then $\rho_{0}^{+} \leqslant \rho^{*}<\rho^{+}$, and thus $f\left(\rho_{0}^{+}\right)$ $=\left(a^{-} / a^{+}\right) f\left(\rho^{-}\right) \leqslant f\left(\rho^{+}\right)$. In either case, we have $d x / d t \geqslant 0$, and thus Eq. (18) is satisfied. Finally, it can be similarly verified that either Eq. (17) or (18) will be violated for any other choice of the pair $\left(\rho_{0}^{-}, \rho_{0}^{+}\right)$, except in two trivial cases. These are $a^{-} f\left(\rho^{-}\right)=a^{+} f\left(\rho^{*}\right)$ for $p \in S_{1}$ and $a^{-} f\left(\rho^{-}\right)=a^{+} f\left(\rho^{+}\right)$for $p$ $\in S_{4}$. In these two cases, the alternatives are $\left(\rho_{0}^{-}, \rho_{0}^{+}\right)$ $=\left(\tilde{\rho}^{-}, \rho^{*}\right)$ and $\left(\rho_{0}^{-}, \rho_{0}^{+}\right)=\left(\tilde{\rho}^{-}, \rho^{+}\right)$, where $\tilde{\rho}^{-}$is uniquely defined by $f\left(\widetilde{\rho}^{-}\right)=f\left(\rho^{-}\right)$and $f^{\prime}\left(\tilde{\rho}^{-}\right)<0$. However, both alternatives produce the same shock curve in $D^{-}$that coincides with the interface $x=0$. Therefore, the solution remains unchanged in $D^{-} \cup D^{+}$

For case (b), let $q(\rho)=a^{-} f(\rho)-a^{+} f\left(\rho^{*}\right)$. We have $q\left(\rho^{*}\right)$ $=a^{-} f\left(\rho^{*}\right)-a^{+} f\left(\rho^{*}\right)>0$, and $q\left(\rho_{m}\right)=-a^{+} f\left(\rho^{*}\right)<0$, and thus $\rho_{0}^{-}$ is uniquely determined by Eqs. (15) and (16). In $D^{+}$, the blank fan is filled with a rarefaction wave; and if any shock exists in $D^{-}$, then Eq. (17) is satisfied [see Fig. 1(b) for the case of $p \in \bar{S}_{1}$ ]. Finally, it can be shown that any other choice of $\left(\rho_{0}^{-}, \rho_{0}^{+}\right)$must violate Eq. (17) or (18).

From the proof of the theorem, we can also obtain the flux at the interface, which is denoted by $\hat{f}=a^{-} f\left(\rho_{0}^{-}\right)=a^{+} f\left(\rho_{0}^{+}\right)$, where $\rho_{0}^{-}$or $\rho_{0}^{+}$has been already specified for each case. For the general case in which $b(x)$ is not constant, we write Eq. (11) in the form

$$
(a \rho)_{t}+(a f(\rho, b))_{x}=0, \quad f(\rho, b)=\rho u_{e}(\rho, b),
$$

and the data of the Riemann problem (12) are extended to include 


$$
b(x)= \begin{cases}b^{-}, & x \in R^{-}, \\ b^{+}, & x \in R^{+} .\end{cases}
$$

Accordingly, we only need to alter these results to $\hat{f}$ $=a^{-} f\left(\rho_{0}^{-}, b^{-}\right)=a^{+} f\left(\rho_{0}^{+}, b^{+}\right)$. The rule for the extension is simply that $b^{-}$and $b^{+}$always accompany $a^{-}$and $a^{+}$, respectively, wherever they appear.

The results are summarized in the following corollary.

Corollary. From the exact Riemann solver for Eqs. (12), (19), and (20), the flux at the interface $x=0$ can be specified by

$$
\hat{f}\left(a^{-}, b^{-}, \rho^{-}, a^{+}, b^{+}, \rho^{+}\right)=\min \left(f^{-}, f^{+}\right),
$$

where

$$
\begin{aligned}
& f^{-}= \begin{cases}a^{-} f\left(\rho^{-}, b^{-}\right), & \text {if } \rho^{-}<\rho^{*}, \\
a^{-} f\left(\rho^{*}, b^{-}\right), & \text {otherwise, }\end{cases} \\
& f^{+}= \begin{cases}a^{+} f\left(\rho^{+}, b^{+}\right), & \text {if } \rho^{+}>\rho^{*}, \\
a^{+} f\left(\rho^{*}, b^{+}\right), & \text {otherwise, }\end{cases}
\end{aligned}
$$

and $f$ can be any strictly concave function of $\rho$.

\section{GODUNOV-TYPE NUMERICAL SCHEMES}

In the following discussion, the dimensionless variables are applied by scaling. Given that the spatial domain of the highway section $(0, L)$ in the analysis, and $u_{m}$ $=\max _{x} b(x), \rho, b, u, x, t$ and $\tau$ are replaced by $\rho \rho_{m}, b u_{m}, u u_{m}, x L, t L / u_{m}$, and $\tau L / u_{m}$, respectively. Correspondingly, the spatial domain $(0, L)$ is scaled to $(0,1)$, and the range of the density $\rho$ becomes $[0,1]$. In the following, the Godunov-type numerical schemes are designed that is based on the Riemann solver using corollary (21) in Sec. III (see Ref. [40] for details of the algorithmic design).

Let $I_{j}=\left(x_{j-1 / 2}, x_{j+1 / 2}\right)$ be the cells that are associated with the discretization of $(0,1), j=1, \ldots, K, x_{1 / 2}=0$, and $x_{K+1 / 2}$ $=1$. In each cell, the node is located at $x_{j}=0.5\left(x_{j-1 / 2}+x_{j+1 / 2}\right)$, and the cell length is $\Delta x_{j}=x_{j+1 / 2}-x_{j-1 / 2}$. To provide the flux values at the spatial boundaries, two additional cells $I_{0}$ $=\left(x_{-1 / 2}, x_{1 / 2}\right)$ and $I_{K+1}=\left(x_{K+1 / 2}, x_{K+3 / 2}\right)$ are considered.

\section{A. Scheme for an extended LWR model}

For the dimensionless variables, the model equation remains the same as it is in Eq. (3) or (19). We specify the model as the following initial value problem (IVP):

$$
\begin{gathered}
(a \rho)_{t}+(a f(\rho, b))_{x}=0, \\
\rho(x, 0)=\rho_{0}(x) .
\end{gathered}
$$

In $I_{j}$, let $a(x)$ be approximated by $a_{j}=a\left(x_{j}\right), b(x)$ be approximated by $b_{j}=b\left(x_{j}\right)$, and $\rho(x, t)$ be approximated by $\rho_{j}(t)$. Then, from the conservation of the total density $a(x) \rho(x, t)$, we have

$$
\rho_{j}(t)=\frac{1}{a_{j} \Delta x_{j}} \int_{I_{j}} a(x) \rho(x, t) d x .
$$

Integrating Eq. (22) with respect to $x$ over $I_{j}$ gives the exact form

$$
a_{j} \Delta x_{j} \frac{\rho_{j}(t)}{d t}+\left.a(x) f(\rho(x, t), b(x))\right|_{x_{j-1 / 2}} ^{x_{j+1 / 2}}=0 .
$$

As the two states in $I_{j}$ and $I_{j+1}$ constitute a local Riemann problem, the flux at the cell interface $\left.a(x) f(\rho(x, t), b(x))\right|_{x_{j+1 / 2}}$ is approximated by

$$
\hat{f}_{j+1 / 2} \equiv \hat{f}\left(a_{j}, b_{j}, \rho_{j}(t), a_{j+1}, b_{j+1}, \rho_{j+1}(t)\right) .
$$

For a time division $\left\{t^{n}\right\}_{n=0}^{N}$ of $t \in[0, T]$, the Euler forward discretization of Eq. (25) yields

$$
\rho_{j}^{n+1}=\rho_{j}^{n}-r_{j}\left(\hat{f}_{j+1 / 2}^{n}-\hat{f}_{j-1 / 2}^{n}\right), j=1, \ldots, K,
$$

where $\quad \rho_{j}^{n}=\rho_{j}\left(t^{n}\right), r_{j}=\Delta t /\left(a_{j} \Delta x_{j}\right), \hat{f}_{j+1 / 2}^{n}$ $=\hat{f}\left(a_{j}, b_{j}, \rho_{j}^{n}, a_{j+1}, b_{j+1}, \rho_{j+1}^{n}\right)$, and $\Delta t=t^{n+1}-t^{n}$. The initial value $\rho_{j}^{0}=\rho_{j}(0)$ is computed by Eqs. (23) and (24), and the function $\hat{f}$ is governed by Eq. (21) because $f^{\prime \prime}(\rho)<0$. Note that this numerical scheme is applicable for any strictly concave function $f(\rho, b)$ of $\rho$.

\section{B. Scheme for an extended higher-order model}

For the EHO model, we adopt the linear speed-density relationship

$$
u_{e}(\rho)=b(1-\rho), \quad 0 \leqslant b \leqslant 1,
$$

from which we obtain

$$
f(\rho)=b \rho(1-\rho),
$$

and $\rho^{*}=0.5$. The consideration of the nonlinear speed-density relationship in the EHO model requires special treatment of the conservation form of the model, which will be investigated in a future study. For the linear speed-density relationship, we define the conservation form of Eq. (9), which together with Eq. (1) constitutes the following system of equations:

$$
(a \rho)_{t}+(a \rho u)_{x}=0
$$

$$
\begin{aligned}
\left(\frac{a u}{b}\right)_{t} & +\left\{0.5 a b\left[\rho^{2}+\left(\frac{u}{b}\right)^{2}-1\right]\right\}_{x} \\
& =0.5 a^{2}\left(1-\rho+\frac{u}{b}\right)\left(1-\rho-\frac{u}{b}\right)\left(\frac{b}{a}\right)^{\prime}+\frac{a\left(U_{e}-u\right)}{b \tau} .
\end{aligned}
$$

The system can be made identical to Eq. (22) in the distribution sense by setting $u=u_{e}(\rho)$ and choosing $U_{e}$ in Eq. (10) such that $\tilde{\tau}=\infty$, which means that Eqs. (29) and (30) reduce to Eq. (22) exactly. In this definition, the features that are inherited from the ELWR model in the formulation will take effect, such as the consideration of stability (see Sec. II B). 
Obviously, these retain the linear transformation of variables (or diagonalization)

$$
\rho=0.5(v-w), \quad u / b=-0.5(w+v),
$$

which is undertaken mostly for the characteristic decomposition on the left-hand side. From Eq. (31), Eqs. (29) and (30) become the equivalent system

$$
(a v)_{t}+(a g(v, b))_{x}=-0.5 a^{2}(1-v)(1+w)\left(\frac{b}{a}\right)^{\prime}-\frac{a\left(U_{e}-u\right)}{b \tau},
$$

$(a w)_{t}+(a g(w, b))_{x}=-0.5 a^{2}(1-v)(1+w)\left(\frac{b}{a}\right)^{\prime}-\frac{a\left(U_{e}-u\right)}{b \tau}$,

where the function $g(v, b)=0.5 b\left(1-v^{2}\right)$. We note that Eqs. (32) and (33) are actually the characteristic equations.

The model is also considered as an IVP with the initial values that are determined by Eq. (31), i.e., $v_{0}(x)=\rho(x, 0)$ $-u(x, 0) / b(x)$ and $w_{0}=-\rho(x, 0)-u(x, 0) / b(x)$. Because the convective parts (the left-hand side) of Eqs. (32) and (33) are analogous to that of Eq. (22), they can be approximated in a similar manner. Denote by $R$ the right-hand side of Eqs. (32) and (33). The numerical scheme of system (32) and (33) is then given by

$$
\begin{gathered}
v_{j}^{n+1}=v_{j}^{n}-r_{j}\left(\hat{g}_{j+1 / 2}^{n}-\hat{g}_{j-1 / 2}^{n}\right)+r_{j} \Delta x_{j} R_{j}^{n}, \\
w_{j}^{n+1}=w_{j}^{n}-r_{j}\left(\widetilde{g}_{j+1 / 2}^{n}-\widetilde{g}_{j-1 / 2}^{n}\right)+r_{j} \Delta x_{j} R_{j}^{n}, \\
v_{j}^{0}=\frac{1}{a_{j} \Delta x_{j}} \int_{I_{j}} a(x) v_{0}(x) d x, w_{j}^{0}=\frac{1}{a_{j} \Delta x_{j}} \int_{I_{j}} a(x) w_{0}(x) d x, \\
j=1, \ldots, K,
\end{gathered}
$$

where $\quad \hat{g}_{j+1 / 2}^{n}=\hat{g}\left(a_{j}, b_{j}, v_{j}^{n}, a_{j+1}, b_{j+1}, v_{j+1}^{n}\right) \quad$ and $\quad \widetilde{g}_{j+1 / 2}^{n}$ $=\hat{g}\left(a_{j}, b_{j}, w_{j}^{n}, a_{j+1}, b_{j+1}, w_{j+1}^{n}\right)$. Because $g^{\prime \prime}(v)<0$, the function $\hat{g}$ is determined by Eq. (21), where $f$ is replaced by $g$. Moreover, we write that $c=b / a$ and approximate $c_{j}^{\prime}$ $=\left[c\left(x_{j+1 / 2}\right)-a\left(x_{j-1 / 2}\right)\right] / \Delta x_{j}$. However, if $c(x)$ is discontinuous at $x_{j+1 / 2}$, then we further approximate

$$
c\left(x_{j+1 / 2}\right)=\alpha_{j} c_{j}+\left(1-\alpha_{j}\right) c_{j+1},
$$

where $\alpha_{j}=\Delta x_{j+1} /\left(\Delta x_{j}+\Delta x_{j+1}\right)$.

We note that schemes (26) and (34) can be made identical by setting $u=u_{e}$ and $\tilde{\tau}=\infty$. This consequence was anticipated due to the conservation form of Eq. (30). Even for the same setting, the numerical result of Eq. (34) would be very different from that of Eq. (26) and would be only loosely bounded (e.g., $\rho<0$ or $\rho>1$ ) if the conservation were defined otherwise.

\section{Characteristics and computational Boundaries}

To finalize schemes (26) and (34), additional nodal values at $x=x_{0}$ and $x=x_{K+1}$ are needed. For initial value problems, these nodal values are given by exact (and usually smooth) solutions, which are based on the parallel characteristic propagation from the initial values. This is a difficult problem for the general case, and thus in the following discussion, a common form of initial data is considered. Note that the density $\rho$ of the ELWR model must be identical to that of the EHO model if $u=u_{e}(\rho)$ and $\tilde{\tau}=\infty$ are set. Therefore, we only need to discuss the latter case.

First, we study the propagation behavior under the assumption that $a(x)$ and $b(x)$ are constant. This implies that $b=1$ and $g(w) \equiv g(w, b)=0.5\left(1-w^{2}\right)$, and that the characteristic equations (32) and (33) become

$$
\begin{aligned}
& v_{t}+g(v)_{x}=-\tau^{-1}\left(U_{e}-u\right), \\
& w_{t}+g(w)_{x}=-\tau^{-1}\left(U_{e}-u\right),
\end{aligned}
$$

where $\tau^{-1}\left(U_{e}-u\right)=\tau^{-1}(1+w)+\widetilde{\tau}^{-1}\left(\widetilde{u}_{e}-u_{e}\right)$, according to Eqs. (10) and (27). Correspondingly, we define the characteristics and thus obtain for the 1 field

$$
\begin{aligned}
\frac{d x}{d t} & =g^{\prime}(v), \quad x(0)=\xi, \quad \frac{d v}{d t}=-\tau^{-1}\left(U_{e}-u\right), \quad v(x(0), 0) \\
& =v_{0}(\xi)
\end{aligned}
$$

and for the 2 field,

$$
\begin{aligned}
\frac{d x}{d t} & =g^{\prime}(w), \quad x(0)=\eta, \quad \frac{d w}{d t}=-\tau^{-1}\left(U_{e}-u\right), \quad w(x(0), 0) \\
& =w_{0}(\eta) .
\end{aligned}
$$

Here, note that $g^{\prime}(v)=-v \leqslant-w=g^{\prime}(w)$. In general, Eq. (37) or (38) cannot be solved explicitly. Suppose that $v_{0}(\xi)\left(=v_{0}\right)$ and $w_{0}(\eta)\left(=w_{0}\right)$ are constant. In this case, the solution is smooth, as is shown in the following, so that the final expressions of Eqs. (37) and (38) can be unified. Thus, the deduction of one from the other yields $v-w=v_{0}-w_{0}$ (constant), which further infers $\rho=\rho_{0}\left[=0.5\left(v_{0}-w_{0}\right)\right]$ and $u=\rho_{0}$ $-v$ or $-\left(\rho_{0}+w\right)$. By the proper substitutions in Eqs. (37) and (38), it follows that for solutions,

$$
\begin{aligned}
w= & C e^{-t / \tau}-U_{e}\left(\rho_{0}\right)-\rho_{0}, \quad v=2 \rho_{0}+w, \quad \rho=\rho_{0}, \\
& u=-C e^{-t / \tau}+U_{e}\left(\rho_{0}\right),
\end{aligned}
$$

the field- 1 characteristics are

$$
x=\xi+\left[U_{e}\left(\rho_{0}\right)-\rho_{0}\right] t+C \tau e^{-t / \tau},
$$

and the field- 2 characteristics are

$$
x=\eta+\left[U_{e}\left(\rho_{0}\right)+\rho_{0}\right] t+C \tau e^{-t / \tau},
$$

where $C=U_{e}\left(\rho_{0}\right)-u_{0}$. In Eqs. (39)-(41), indeed, the characteristics of the same field are superposable by translation, so that each propagation of the two families of characteristics is parallel, and a smooth solution is formed.

Applying these results, the left boundary values are acquired by assuming that $v_{0}(\xi)\left(=v_{l}\right)$ and $w_{0}(\eta)\left(=w_{l}\right)$ are constant for $x<X_{1}$. This is indicated in Fig. 2, where the boundary $x=x_{0}$ (with $t<T_{1}$ ) fall into the smooth solution domain $D_{1}=\left\{(x, t) \mid x<X_{1}(t), t \geqslant 0\right\}$. The application of Eq. (39) to this smooth solution region yields 


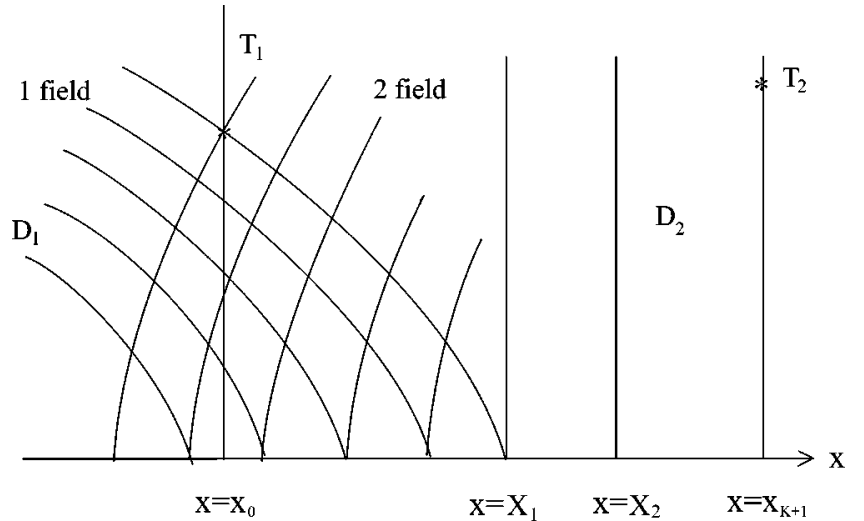

FIG. 2. Obtaining the boundary values for the numerical schemes through the propagation of characteristics.

$$
\begin{aligned}
w_{0}^{n}= & C_{l} e^{-n \Delta t / \tau}-U_{e}\left(\rho_{l}\right)-\rho_{l}, \quad v_{0}^{n}=C_{l} e^{-n \Delta t / \tau}-U_{e}\left(\rho_{l}\right)+\rho_{l}, \\
& C_{l}=U_{e}\left(\rho_{l}\right)-u_{l} .
\end{aligned}
$$

If the propagation speed of the field- 1 characteristics is negative, then $X_{1}(t)$ is the field-1 characteristic curve from $x$ $=X_{1}^{-}$. In this case, $T_{1}$ is the intersection of $x=X_{1}(t)$ and the line $x=x_{0}$, and thus is determined by Eq. (40) such that $\rho_{0}, C, \xi$ and $(x, t)$ are replaced with $\rho_{l}, C_{l}, X_{1}$ and $\left(x_{0}, T_{1}\right)$, respectively. Otherwise, $X_{1}(t)=X_{1}$ and $T_{1}=\infty$.

It is similar to obtain the right boundary values in $x$ $=x_{N+1} \quad$ (with $t<T_{2}$ ), under the assumption that $a(x)$, $v(x, 0)\left(=v_{r}\right)$, and $w(x, 0)\left(=w_{r}\right)$ are constant for $x>X_{2}$. These boundary values read

$$
\begin{aligned}
w_{N+1}^{n}= & C_{r} e^{-n \Delta t / \tau}-U_{e}\left(\rho_{r}\right)-\rho_{r}, \\
& v_{N+1}^{n}=C_{l} e^{-n \Delta t / \tau}-U_{e}\left(\rho_{r}\right)+\rho_{r}, \quad C_{r}=U_{e}\left(\rho_{r}\right)-u_{r},
\end{aligned}
$$

and $T_{2}$ is determined by Eq. (41) such that $\rho_{0}, C, \eta$, and $(x, t)$ are replaced, respectively, with $\rho_{l}, C_{l}, X_{2}$, and $\left(x_{N+1}, T_{2}\right)$. Note that the propagation of the 2 characteristics is always positive.

The boundary conditions of Eqs. (42) and (43) are valid only for $t<T=\min \left(T_{1}, T_{2}\right)$. We emphasize that their validity can be identified by

$$
\begin{aligned}
\rho_{0}^{n}= & 0.5\left(v_{0}^{n}-\right. \\
& \left.w_{0}^{n}\right)=v_{l}-w_{l} \equiv \rho_{l}, \rho_{K+1}^{n}=0.5\left(w_{K+1}^{n}-v_{K+1}^{n}\right)=v_{r}-w_{r} \\
\equiv & \rho_{r},
\end{aligned}
$$

which means that the density $\rho$ remains constant in the two boundaries.

When setting $u=u_{e}(\rho)$ (and $\tilde{\tau}=\infty$ ) in Eqs. (42) and (43), the boundary values of the ELWR model are obtained. Clearly, Eq. (44) also gives these values.

As a summary, some fundamental features of the EHO model are emphasized below. First, Eq. (39) indicates that the velocity $u$ relaxes to the equilibrium state $U_{e}(\rho)$. For this smooth solution, moreover, the propagations are nearly straight lines for large $t$ [Eqs. (40) and (41)]. However, a disturbance that includes changes in $\rho, u, a(x)$, and $b(x)$ usually gives rise to wave breaking (discontinuity) and therefore more complicated propagation behavior. It is well known that the propagation speed (and thus the behavior) of discontinuity depends on the conservation forms of the model equations, which is why the conservative form of Eq. (9) must be given regardless of the physical conservation of a quantity. This reasonably defines the safer propagations because Eqs. (29) and (30) together are consistent with the ELWR model for their conservative parts.

Second, for the case in which $\tilde{\tau}=\infty\left(U_{e}=u_{e}\right.$ or $U_{e}-u=1$ $+w)$, Eqs. (40) and (41) can be solved separately, even with the initial data that $v_{0}(\xi)$ and $w_{0}(\eta)$ are not constant. The solution for Eq. (41) for the 2 field reads

$$
\begin{gathered}
x=\eta+t-\tau\left[1+w_{0}(\eta)\right]\left(1-e^{-t / \tau}\right), \\
w=\left[1+w_{0}(\eta)\right] e^{-t / \tau}-1,
\end{gathered}
$$

which indicates that the propagation is asymptotical to a straight line and $u$ to $u_{e}(\rho)(w \rightarrow-1)$. For wave breaking that is governed by the conservation forms (29) and (30), the whole solution must be asymptotical to that of the ELWR model.

\section{NUMERICAL EXAMPLES}

In this section, the numerical schemes are tested and the asymptotical property of the EHO model to the ELWR model is confirmed by $\tilde{\tau}=\infty$. These demonstrate that the numerical solutions are truly convergent to the analytical solutions (if they are well posed), so that the schemes can be further applied to describe more complicated occurrences, and probably to predict new traffic flow phenomena. With traffic signal example, the control is well represented by the free-flow velocity $b$, and waves are described and explained reasonably in a new way. We specify the parameters $\rho_{m}$ $=0.15$ vehicles $/ \mathrm{m}$ and $u_{m}=\max _{x} b(x)=20 \mathrm{~m} / \mathrm{s}$. The dimensionless variables are used in all figures, and a dimensional variable is always followed by its unit so that they can easily be distinguished.

For simplicity, we set $b(x)=1$ in the first three examples that represent the traffic flow around a bottleneck, as given by the Riemann problem (12). Here, the interface $x=0$ changes to $x=x_{0}$, with $\left(a^{-}, a^{+}\right)=(3,1)$; the simulation time and length are chosen to be $T=240 \mathrm{~s}$ and $L=4000 \mathrm{~m}$ with the division by $\Delta t=0.2$ and $\Delta x_{j}=10 \mathrm{~m}$. For comparison, the EHO model of $\tilde{\tau}=\infty$ is also solved with the same set of parameters, together with the relaxation time $\tau=10 \mathrm{~s}$ the initial speeds $u^{-} \equiv u(x, 0)\left(x<x_{0}\right)$, and $u^{+} \equiv u(x, 0)\left(x>x_{0}\right)$.

We show the results in Figs. 3, 4, and 5, and compare them with the analytical results in Sec. III. In all of these examples, the shock and rarefaction waves that are described by the ELWR model are well captured. For the EHO model, the solution structures become very similar to those of the ELWR model as $t$ increases, which is consistent with the analysis in the previous sections.

In contrast, the EHO model is very different if $\tilde{\tau}$ is finite. As $U_{e}(\rho) \neq u_{e}(\rho)$, its conservative parts [the second and third terms in Eqs. (32) and (33)] conflict with the relaxation, and 

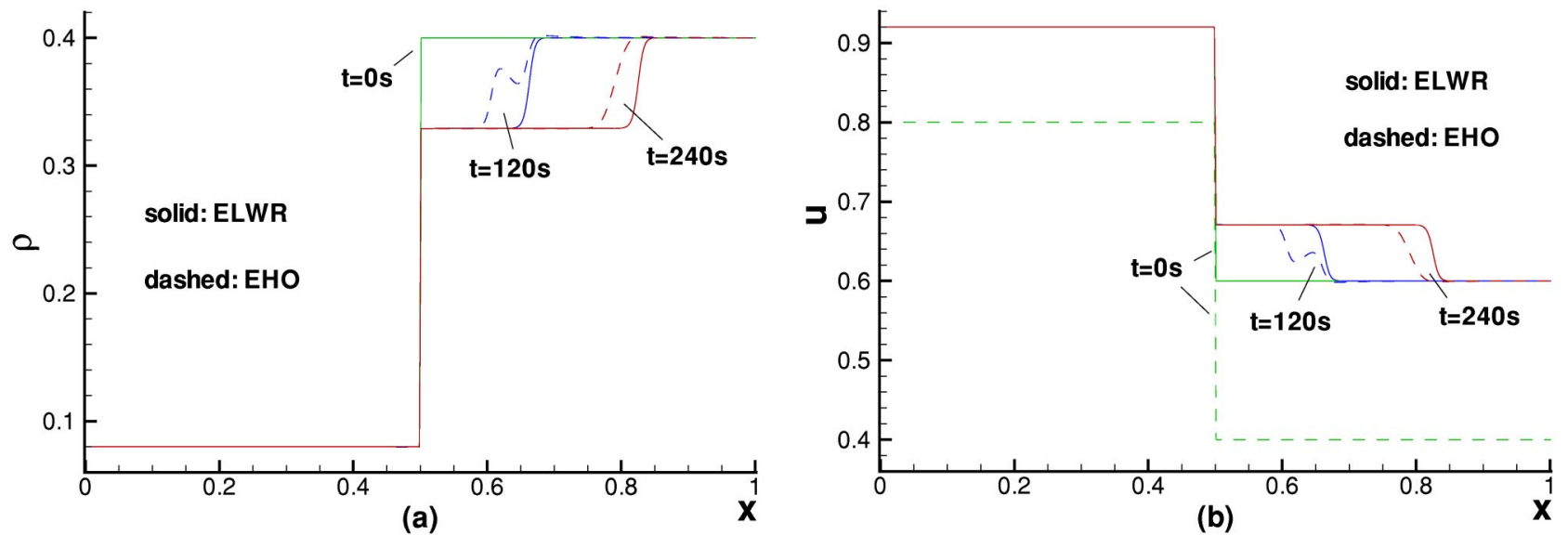

FIG. 3. Traffic flow corresponding to $p \in S_{1}$ in the Riemann problem, with $x_{0}=0.5,\left(\rho^{-}, \rho^{+}\right)=(0.08,0.4)$. The propagation of $\rho^{-}=0.08$ passes through the bottleneck and results in a shock downstream. The solutions of the ELWR and EHO models are compared by setting $\left(u^{-}, u^{+}\right)=(0.8,0.4)$.

thus $u$ is not convergent to $U_{e}(\rho)$. Being nonzero, the fluctuation component of the relaxation term dominates. In this case, the traffic waves are very similar to those in detonation. This feature of the high-order models was discussed in Kerner and Konhauser $[13,14]$, and it was found that a small perturbation to the constant distribution of the velocity and density may give rise to the formation of an traffic platoon that propagates in oscillation or as a prolonged jam or queue. In other words, the solution that is given by Eq. (39) in a homogeneous traffic section is sensitive (unstable or metastable) to the perturbation, and would be magnified if its length were sufficiently large.

In this model, the perturbation is physically related to changes in the traffic capacity. The free-flow speed $b$ is further extended to be a temporal variable to represent changes in the capacity in some special locations. This representation is straightforward in the ELWR model, and is implied in the EHO model by comparing accelerations (5) and (9). The same extension for the number of lanes $a$ could be conducted with similar reasoning, for example, in locations near an ac-

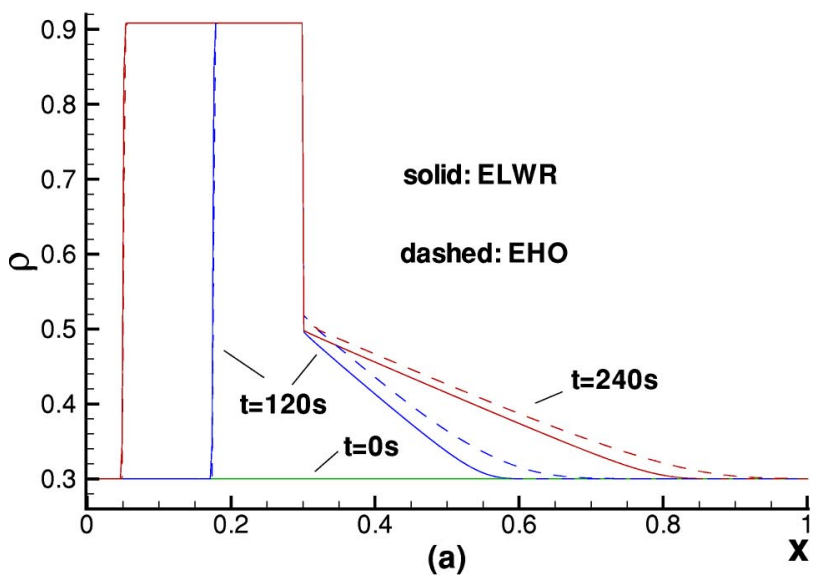

cident, but this is not included. Here, we note that the main motivation is again to demonstrate the robustness of the numerical schemes. For example, it is challenging to apply $b(x, t)$ such that it jumps from the maximum 1 to the minimum 0 , but in the present analysis, the numerical results are physically bounded.

A few examples are simulated by the EHO model below, by choosing [13]

$$
\bar{u}_{e}(\rho, b)=b\left\{\left[1+\exp \left(\frac{\rho-0.25}{0.06}\right)\right]^{-1}-3.72 \times 10^{-6}\right\} .
$$

Recall the previous discussion and refer to Eqs. (9) and (10). It should be noted that a smaller $\tilde{\tau}$ means more conflict between the convective and relaxation effects, and thus a greater likelihood of fluctuation. More precise (and physically relevant) simulation can be made by a comparison with the observed data, following similar ideas and adjusting the parameters.

FIG. 4. Traffic flow corresponding to $p \in \bar{S}_{1}$ in the Riemann problem, with $x_{0}=0.3,\left(\rho^{-}, \rho^{+}\right)=(0.3,0.3)$. The propagation of $\rho^{-}=0.3$ is obstructed by the bottleneck, which results in a shock on the left-hand side and a rarefaction on the right-hand side. The solutions of the ELWR and EHO models are compared by setting $u^{-}=u^{+}=0.9$. 

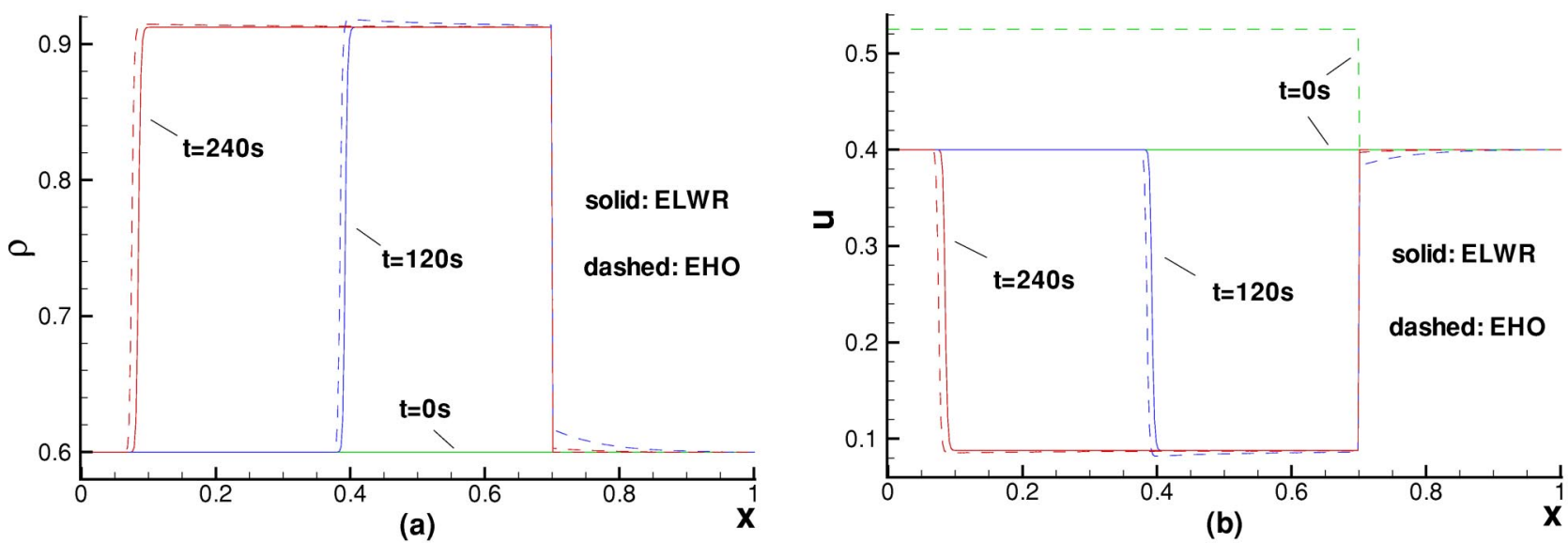

FIG. 5. Traffic flow corresponding to $p \in \bar{S}_{3}$ in the Riemann problem, with $x_{0}=0.7,\left(\rho^{-}, \rho^{+}\right)=(0.6,0.6)$. The propagation of $\rho^{+}=0.6$ passes through the bottleneck and results in a shock upstream. The solutions of the ELWR and EHO models are compared by setting $\left(u^{-}, u^{+}\right)$ $=(0.525,0.4)$.

Consider a traffic flow initially with $\rho_{0}(x)$ being constant and $u_{0}(x)=u_{e}\left(\rho_{0}(x)\right)$. It will converge to a constant flow according to Eq. (39). However, we perturb near location $x$ $=x_{p}$ by changing the values of $b(x, t)$. The computational length is chosen to be sufficiently large, i.e., $L=120000 \mathrm{~m}$, so that the boundary values (42) and (43) are applicable to a long simulation. The computational region is divided by $\Delta x=100 \mathrm{~m}$ and $\Delta t=1 \mathrm{~s}$.

In Fig. 6, these data suggest that the flow should be forbidden near $x_{p}=0.5$ for a sufficiently long time for a jam to form. After the perturbation is released, the jam (cluster) propagates upstream and Fig. 6(a) shows the density at $t$ $=1800 \mathrm{~s}$. The fundamental diagram or density-flow state $(\rho, q),(q=\rho u)$ is shown in Fig. 6(b), which is also compared with the curve $q=\rho U_{e}(\rho)$. Corresponding to states 1 to 3 in Fig. 6(a), the states that are represented in Fig. 6(b) show the deceleration path when vehicles enter the jam and the acceleration path when they leave the jam. The shape of the cluster remains almost unchanged because the in-flow and outflow (both represented by state 1) are approximately the same.

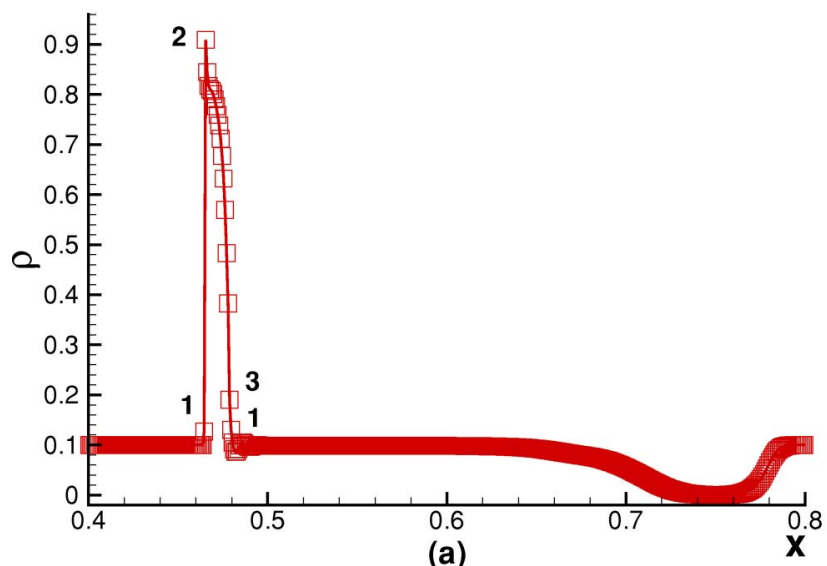

In Fig. 7, the perturbation is made periodically near $x_{p}$ $=0.2$, such that $b=1$ reduces to $b=0.5$ for $60 \mathrm{~s}$ in each period of 200 s. A series of clusters are observed in Fig. 7(a), and the fundamental diagram [Fig. 7(b)] shows the mechanism of the formation in a similar fashion.

In Fig. $8, \rho_{0}(x)$ is relatively large, and $b=1$ reduces to $b$ $=0.5$ near $x_{p}=0.4$ for $3 \mathrm{~s}$ in each period of $20 \mathrm{~s}$. The length of the first jam [Fig. 8(a)] is increasing because the in-flow is much larger than the out-flow. These two flows correspond to the heights at $\rho=0.2$ and $\rho \approx 0.1$ in Fig. 8(b), respectively. In addition, oscillations that propagate downstream are observed, which correspond to the high flow states between $\rho$ $=0.1$ and $\rho=0.5$ in Fig. 8(b).

The periodic boundary condition has been widely used in traffic flow problems $[13,14]$. The road section $[0, L]$ is a circular track, and thus the number of vehicles is fixed in the interval. Note that a propagation over one of the two boundaries is connected by the other boundary. In application, the downstream-propagating oscillations in Fig. 8(a) would be eventually absorbed in the first jam, and the length of the jam would then be narrowed because the forthcoming flow is smaller than the out flow. Because $\rho_{0}(x)$ is small, some of the

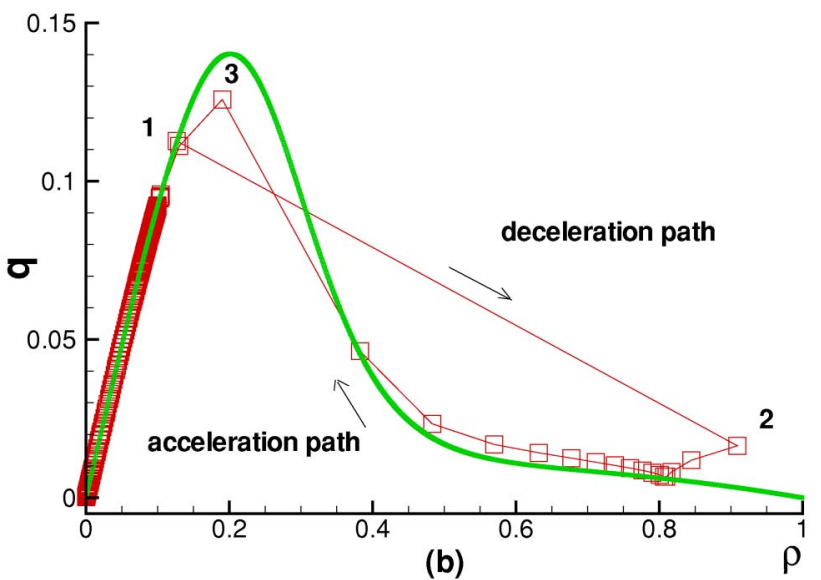

(b)

FIG. 6. Traffic flow state at $t=1800 \mathrm{~s}$, as a consequence of a disturbance to the constant initial data $\rho_{0}(x)=0.1$ and $u_{0}(x)=u_{e}\left(\rho_{0}(x)\right)$, by setting $b(x, t)=0$ for $0.499<x<0.5$ and $1 \mathrm{~s}<t \leqslant 501 \mathrm{~s}$, and $b(x, t)=1$ for others, and with $\tau=1 \mathrm{~s}, \tilde{\tau}=1.04 \tau$. (a) Distribution of density $\rho$; (b) fundamental diagram compared with $q=\rho U_{e}(\rho)$. 

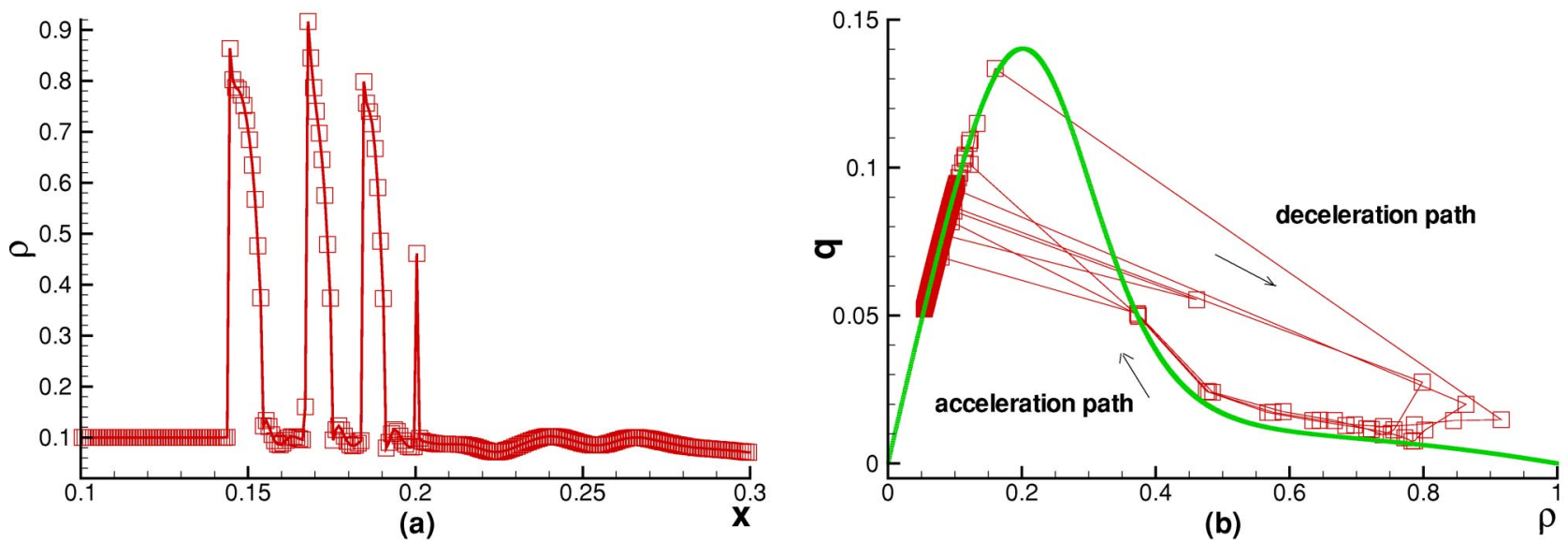

FIG. 7. Traffic flow state at $t=3600 \mathrm{~s}$, as a consequence of a disturbance to the constant initial data $\rho_{0}(x)=0.1$ and $u_{0}(x)=u_{e}\left(\rho_{0}(x)\right)$ by setting $b=0.5$ for $0.199<x<0.2,1 \mathrm{~s}<t-200[t / 200] \leqslant 61 \mathrm{~s}$, and $b=1$ for others, and with $\tau=1 \mathrm{~s}$ and $\tilde{\tau}=1.04 \tau$. (a) Distribution of density $\rho$; (b) fundamental diagram compared with $q=\rho U_{e}(\rho)$.

jams in Fig. 7(a) are expected to die out. These results are confirmed by computation, which is not shown.

Applying the periodic boundary conditions, we show an interesting example in Fig. 9, for which $L=20000 \mathrm{~m}, \Delta x$ $=100 \mathrm{~m}, \Delta t=0.5 \mathrm{~s}$, and the perturbation $b=0$ lasts for only $10 \mathrm{~s}$ near $x_{p}=0.5$. In addition to the formation of a upstreampropagating jam that is similar to those in Figs. 7(a) and 8(a), there are oscillations that propagate downstream [Fig. 9(a)]. Between $x=0.60$ and 0.65 , for example, they are hysteretic with a high frequency at $t=500 \mathrm{~s}$, and these congested densities have a higher flow [and a higher velocity than suggested by $\left.u=U_{e}(\rho)\right]$ that corresponds to the states over the curve $q=\rho U_{e}(\rho)$ in Fig. 9(b). Due to the higher flow in the congested region, the oscillations keep almost stationary, and at $t=700 \mathrm{~s}$ [Fig. 9(c)] they merge into a new jam with states that come much closer to the curve $q=\rho U_{e}(\rho)$ [comparing Figs. 9(b) and 9(d)]. Therefore, the second jam is similar to the first jam in that it is observed to propagate upstream in Fig. 9(e). The third jam is formed at approximately $x=0.75$ by a similar mechanism, and begins to propagate upstream

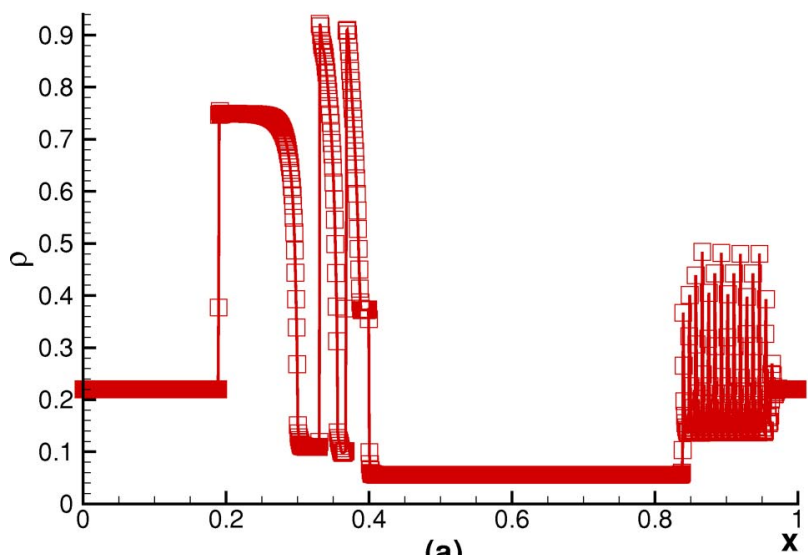

(a) when the propagation of the first jam meets the first hump of congested traffic. This first jam continues to propagate and passes through the periodic boundary. It then absorbs all the humps so that the solution eventually evolves into three jams with free flows in between [Fig. 9(e)]. These three jams propagate with approximately unchanged shapes and the same speed, and are known as traveling waves.

This example is typical of metastability in traffic, and a reasonable explanation might be that the initial traffic state is so dense that unstable blow-ups and oscillations come up in a perturbation as a sacrifice to maintain free traffic in the surrounding area. These blow-ups and oscillations will eventually evolve into another stable state that is characterized by upstream-propagating jams. In our numerical test, this conclusion is generally true if the periodic condition is applied (see Refs. [13,14,39] for similar accounts).

We can take advantage of the extended models and schemes to simulate signal traffic. Specifically, the signal is controlled by setting $b=0$ for red and $b=1$ for green. In this way, the physical meaning is much clearer, and the evolution may be taken to be any lengthy period. Moreover, a periodic

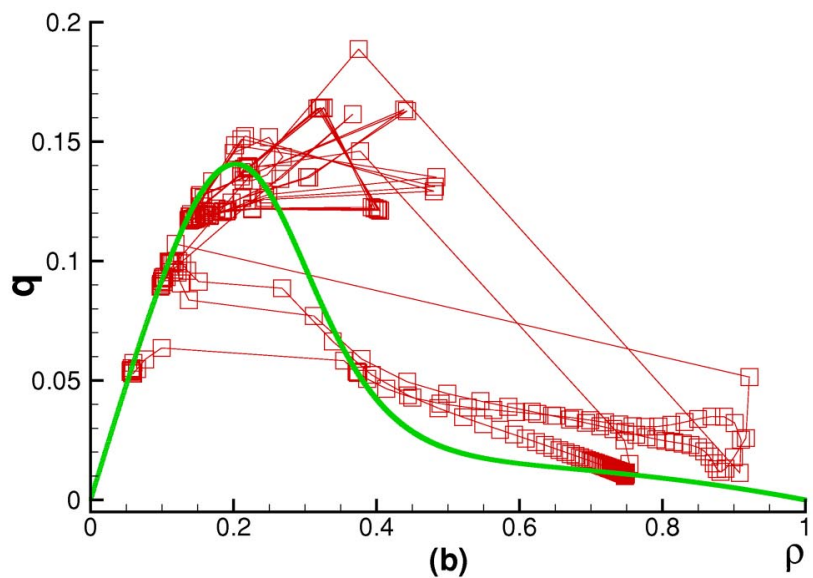

(b)

FIG. 8. Traffic flow state at $t=5400 \mathrm{~s}$, as a consequence of a disturbance to the constant initial data $\rho_{0}(x)=0.22$ and $u_{0}(x)=u_{e}\left(\rho_{0}(x)\right)$ by setting $b=0.5$ for $0.399<x<0.4$ and $1 \mathrm{~s}<t-20[t / 20] \leqslant 4 \mathrm{~s}$, and $b=1$ for others, and with $\tau=10 \mathrm{~s}, \tilde{\tau}=1.06 \tau$. (a) Distribution of density $\rho$; (b) fundamental diagram compared with $q=\rho U_{e}(\rho)$. 

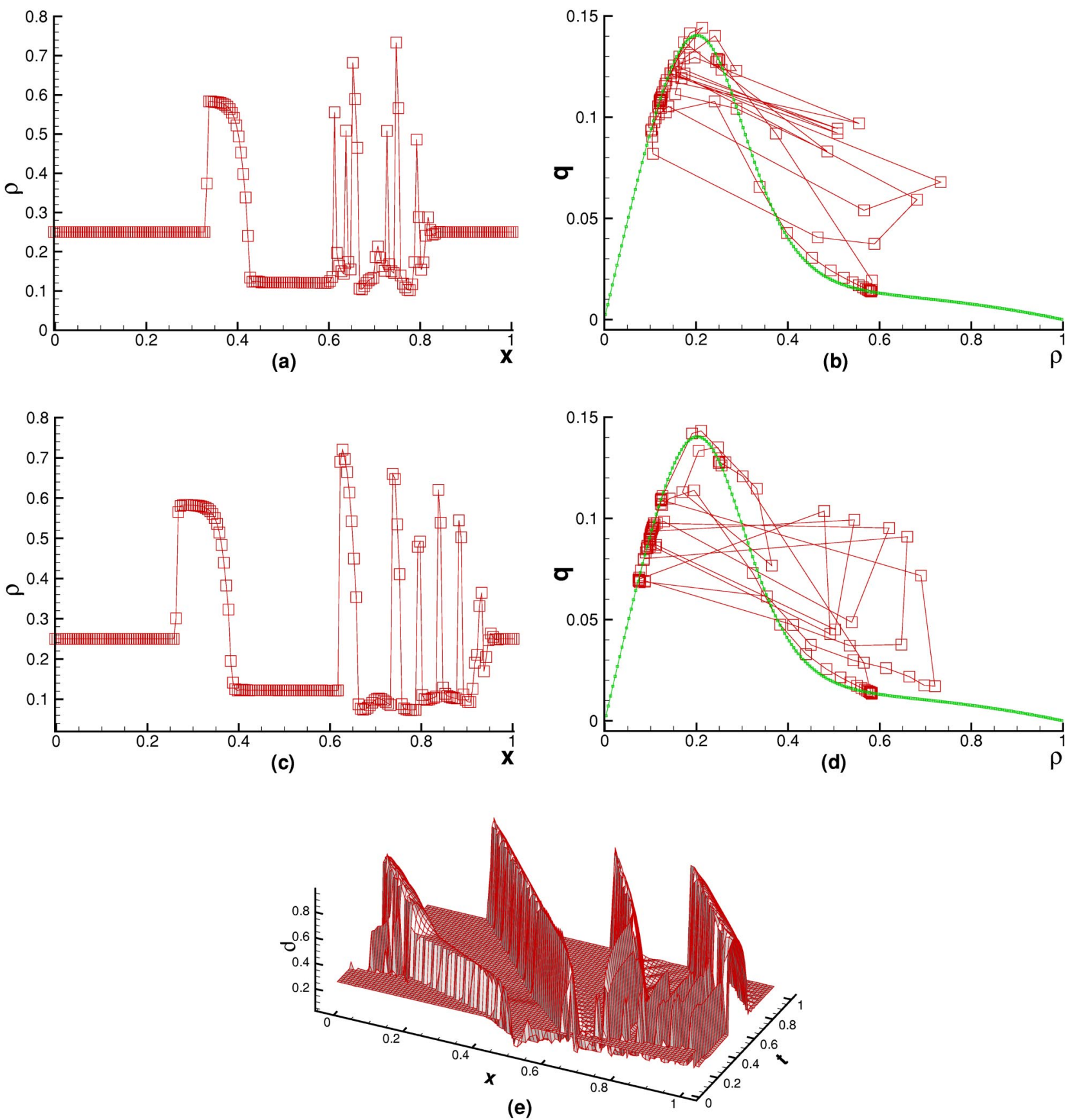

FIG. 9. Traffic flow state as a consequence of a disturbance to the constant initial data $\rho_{0}(x)=0.25$ and $u_{0}(x)=\tilde{u}_{e}\left(\rho_{0}(x)\right)$ by setting $b$ $=0$ for $0.494<x<0.5$ and $1 \mathrm{~s}<t<11 \mathrm{~s}$, and $b=1$ for others, and with $\tau=3 \mathrm{~s}, \tilde{\tau}=1.05 \tau$. (a) and (b) $t=500 \mathrm{~s}$; (c) and (d) $t=700 \mathrm{~s}$; (e) evolution for $t \in[0 \mathrm{~s}, 4800 \mathrm{~s}]$.

boundary condition is applied by which we imply that the evolutions in the neighboring sections are the same. One example for this is shown in Fig. 10, which is simulated by the EHO model, where the red signal is taken to be $30 \mathrm{~s}$ in every $60 \mathrm{~s}$ and near $x=0.8$ for $L=2000 \mathrm{~m}$. The result shows a flow that consists of free, congested, and jamming traffic in fixed locations.

\section{CONCLUSIONS}

We have presented two types of models that describe the traffic flow on a highway with variable lanes and free-flow velocities, which are extended from the LWR and higher order models. To approximate the numerical flux $f(u(x, t), a(x))$, or specifically $a(x) f(\rho(x, t), b(x))$ in our case, the Riemann problem is discussed. Based on the wave breaking, we have developed efficient Godunov-type numerical schemes for the models. The traffic flow across a bottleneck (lane drop) has been simulated and compared with the exact Riemann solution. The results demonstrate the effectiveness of the numerical schemes, based on which we can further study inhomogeneous freeway traffic. 


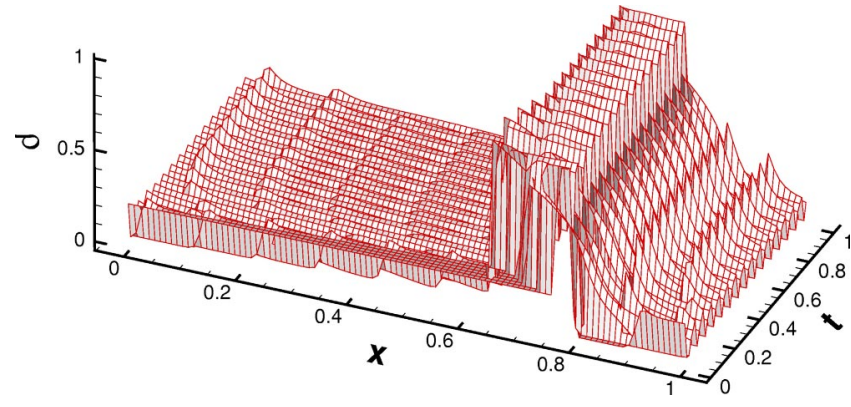

FIG. 10. Traffic flow states for $t \leqslant 900 \mathrm{~s}$, as a consequence of a disturbance to the constant initial data $\rho_{0}(x)=0.2$ and $u_{0}(x)$ $=\tilde{u}_{e}\left(\rho_{0}(x)\right)$ by setting $b=0$ for $0.795<x<0.8$ and $1 \mathrm{~s}<t$ $-60[t / 60]<31 \mathrm{~s}$, and $b=1$ for others, and with $r=10 \mathrm{~s}, \tilde{\tau}$ $=1.08 \tau, L=2000 \mathrm{~m}, \Delta x=10 \mathrm{~m}, \Delta t=0.2 \mathrm{~s}$.

In another numerical simulation with the EHO model where $U_{e}$ is closer to $\widetilde{u}_{e}$, free, congested, and jamming traffic flows are all captured. They appear as a consequence of perturbations to the constant flow with a relatively higher density. It is physically meaningful that the perturbation is made by the change in the free-flow velocity in special locations that represent the drop in traffic capacity and the recovery. For the application of the periodic boundary condition, the evolution typically shows the metastablity for a denser initial distribution in that it includes unstable oscillations (congested traffic), but eventually becomes regular jams. From the fundamental diagram, it can be seen that the propagation of these upstream-propagating jams is governed by the LWR model, because all of the states in the cluster fall closely with the curve $q=\rho U_{e}(\rho)$, and thus the relaxation term is nearly zero. This explains why the propagation can be stable. This property is not mentioned, but will be treated by a rigorous account in a future study.

In the formulation that includes the definition of conservation forms (with linear function $u_{e}=1-\rho$ ), we note that the EHO model is so closely related to the ELWR model that their convective parts are consistent. This guarantees that the EHO model shares a similar numerical flux function, which is given by Eq. (21). By extension, $\Delta x$ in Eq. (6) might be alternatively chosen, or another formulation could be used. However, for sharp changes in the lane number $a$ and the free-flow velocity $b$, the difficulty in obtaining a numerical solution must be overcome, unless the exact (or an approximate high-resolution) Riemann solver for the system is developed. In general, obtaining such a Riemann solver would be a challenge for these systems, and future research could concentrate on a more complicated formulation of EHO models. We should also note that the problem of negative velocity sometimes occurs with the EHO model in our numerical analysis, due to the isotropic viscosity that is inherent in higher-order models [11]. Therefore, the extension of the method to anisotropic higher-order models should also be investigated.

\section{ACKNOWLEDGMENTS}

We wish to thank Dr. Xiao-Chuan Wang and the anonymous referees for their helpful and constructive comments and suggestions on the earlier draft of this paper. This work was supported by grants from the National Natural Science Foundation of China (Grant Nos. 10472064,10371118), the China Postdoctoral Science Foundation (Grant No. 2003034254), and the Hong Kong Research Grants Council (Grant No. HKU7031/02E).
[1] M. J. Lighthill and G. B. Whitham, Proc. R. Soc. London, Ser. A 229, 317 (1955).

[2] P. I. Richards, Oper. Res. 4, 42 (1956).

[3] H. J. Payne, Mathematical Methods in Public Systems, edited by G.A.Bekey (Simulation Council, La Jolla, CA, 1971), Vol. 1, p. 51.

[4] G. B. Whitham, Linear and Nonlinear Waves (John Wiley and Sons, New York, 1974).

[5] R. D. Kuhne, in Proceedings of the 9th International Symposium on Transportation and Traffic Theory, edited by J. Volmuller and R. Hamerslag (VNU Science Press, Utrecht, 1984), pp. 21-42.

[6] P. Ross, Transp. Res., Part B: Methodol. 22, 421 (1988).

[7] P. G. Michalopoulos, P. Yi, and A. S. Lyrintzis, Transp. Res., Part B: Methodol. 27, 315 (1993).

[8] G. F. Newell, Transp. Res., Part B: Methodol. 27, 281 (1993).

[9] G. F. Newell, Transp. Res., Part B: Methodol. 27, 289 (1993).

[10] G. F. Newell, Transp. Res., Part B: Methodol. 27, 305 (1993).

[11] C. F. Daganzo, Transp. Res., Part B: Methodol. 29, 277 (1995).

[12] C. F. Daganzo, Transp. Res., Part B: Methodol. 29, 261 (1995).
[13] B. S. Kerner and P. Konhauser, Phys. Rev. E 48, R2335 (1993).

[14] B. S. Kerner and P. Konhauser, Phys. Rev. E 50, 54 (1994).

[15] D. Helbing, Phys. Rev. E 51, 3164 (1995).

[16] D. Helbing, in Traffic and Granular Flow, edited by D. E. Wolf, M. Schreckenberg, and A. Bachem (World Scientific, Singapore, 1996), pp. 87-104.

[17] H. M. Zhang, Transp. Res., Part B: Methodol. 32B, 485 (1998).

[18] H. M. Zhang, Transp. Res., Part B: Methodol. 35337 (2001).

[19] A. Aw and M. Rascle, SIAM J. Appl. Math. 60, 916 (2000).

[20] S. C. Wong and G. C. K. Wong, Transp. Res., Part B: Methodol. 36, 683 (2002).

[21] G. C. K. Wong and S. C. Wong, Transp. Res., Part A 36, 827 (2002).

[22] M. P. Zhang, C.-W. Shu, G. C. K. Wong, and S. C. Wong, J. Comput. Phys. 191, 639 (2003).

[23] Zhang Peng and Liu Ru-Xun, Chin. J. Comput. Phys. 18, 229 (2001).

[24] D. Helbing, Phys. Rev. E 55, 5498 (1997).

[25] D. Helbing, Phys. Rev. E 59, 6328 (1999).

[26] D. Helbing, Verkehrsdynamik [Traffic Dynamics] (Springer, 
Berlin, 1997).

[27] G. F. Newell, in Proceedings of the 14th International Symposium on Transportation and Traffic Theory, edited by A. Ceder (Pergamon, New York, 1999), pp. 125-146.

[28] Zhang Peng and Liu Ru-Xun, Chin. J. Comput. Phys. 19, 142 (2002)

[29] Zhang Peng and Liu Ru-Xun, Chin. J. Comput. Phys. 20, 130 (2003).

[30] P. Zhang and R. X. Liu, J. Comput. Appl. Math. 156, 1 (2003).

[31] P. Zhang and R. X. Liu, J. Comput. Appl. Math. 176, 105 (2005).

[32] P. Zhang and R. X. Liu, Numer. Methods Partial Differ. Equ. 21(1), 80 (2005).

[33] B. Cockburn, C. Johnson, C. -W.Shu, and E. Tadmor, in Advanced Numerical Approximation of Nonlinear Hyperbolic
Equations, edited by A.Quarteroni, Springer Lecture Notes in Mathematics, Vol. 1697 (Springer, New York, 1998), pp. 151185.

[34] T. Gimse and N. H. Risebro, SIAM J. Math. Anal. 23, 635 (1992).

[35] L. Lin, J. B. Temple and J. Wang, SIAM (Soc. Ind. Appl. Math.) J. Numer. Anal. 32, 824 (1995).

[36] A. Tveito and R. Winther, SIAM J. Sci. Comput. (USA) 16, 320 (1995).

[37] R. A. Klausen and N. H. Risebro, J. Diff. Eqns. 157, 41 (1999).

[38] L. A. Pipes, Transp. Res. 3, 229 (1969).

[39] D. Helbing, Rev. Mod. Phys. 73, 1067 (2001).

[40] E. F. Toro, Riemann Solvers and Numerical Methods for Fluid Dynamics: A Practical Introduction (Springer, Berlin, 1999). 